\title{
Time-Scale Analysis of Malaria Dynamics in Human-Mosquito Population
}

\author{
Kodwo Annan \\ School of Science \& Technology, Georgia Gwinnett College, Lawrenceville, USA
}

Email address:

kannan@ggc.edu

\section{To cite this article:}

Kodwo Annan. Time-Scale Analysis of Malaria Dynamics in Human-Mosquito Population. International Journal of Theoretical and Applied Mathematics. Vol. 3, No. 2, 2017, pp. 88-93. doi: 10.11648/j.ijtam.20170302.17

Received: January 20, 2017; Accepted: February 13, 2017; Published: March 2, 2017

\begin{abstract}
More realistic human-mosquito population mathematical model in which re-infected asymptomatic humans are considered is presented. Six possible time-scale of events for model transition from non-endemic to endemic state are analyzed. Results show that the buildup of the latent asymptomatic humans at steady state is the main dynamics of malaria in the endemic region. This become evident in the time scale of about 1-2 weeks and thus influences the mode of infection in the malaria transmission analysis.
\end{abstract}

Keywords: Malaria Transmission, Timescale Analysis, Mathematical Modeling

\section{Introduction}

Malaria is a vector born disease that can be transmitted to humans through infected anopheles mosquito bites. The parasite's life cycle is well described in literatures $[1,2]$. Symptoms of the disease include vague, anemia, blood stools, convulsion, myalgia, diarrhea, nausea, vomiting and others [3]. The infection can also lead to damage of human organs such as the brain, lungs, kidneys and blood vessels [3, 4]. Extensive research efforts have been focused to the study of the transmission of the malaria parasite between humans and mosquitoes and how to control the disease in the endemic regions [5-7]. Despite substantial progress, it is still a major global problem in terms of morbidity and mortality especially in the endemic regions. In 2015, the World health organization reported 214 million high risk malaria cases and over 438,000 deaths worldwide [8].

The complexity of the disease's zoonotic nature makes it clinically impossible to understand fully the mechanism in which the disease spreads. Clinically, two methods are in use: one which reduces the risk and intensity of malaria and death and another which uses protective antimalarial immunity to reduce the number of parasites in an infected individual. In both methods, the tendency for malaria patients to become resistant to these methods makes many malaria patients temporarily asymptomatic parasite carriage $[9,10]$. Although there is no standard definition for "asymptomatic" malaria infections, it is generally accepted to be malarial parasitemia of any density, in the absence of fever or other acute symptoms in individuals [7]. These infected individuals sustain malaria overtime and so are important source of malaria transmission and therefore a major obstacle to eradication programs. Ogutu et al. [11], reported in their that a large proportion of malaria infections are with microscopy-detection level as high as 39 percent on children under 10 years old in endemic regions. Based on findings they hypothesized without testing that a significant reduction of the malaria parasite pool could be obtained through the treatment of the asymptomatic class in endemic population.

Some recent papers have used mathematical modeling approach to provide more insight of the effects of asymptomatic parasite carriage on malaria transmission in human and mosquito populations [2, 12-15]. These models have played important roles in influencing intervention strategies for preventing and controlling the transmission of malaria. However, of these papers, only one [2] made an attempt to study the evolution of malaria based on the reproduction number $\left(R_{0}\right)$ and a reasonable timescale to demonstrate the existence and effects of the asymptomatic group in malaria dynamics. In their paper, Annan et al. analyzed the transition model by adducing sufficient conditions to show that malaria free state in which asymptomatic group is present is locally and asymptotically stable if $R_{0}<1$ and unstable for $R_{0}>1$. In addition, they 
performed a preliminary time scale analysis to show that the asymptomatic individuals at steady state may be one of the main force in transmitting malaria in endemic regions.

This paper explores this notion by providing detailed timescale analysis of the model formulated in [2]. The six main timescales predicted by the model are used with appropriate rescaling to explore the dynamics of malaria from early incidence (i.e. when a small infected mosquitoes are introduced into human-mosquito population) to endemic state. Finding the time to develop this asymptomatic status by malaria patients in an endemic state may be essential in planning control and eradication strategies.

We organize the rest of the paper as follows. Dimensionless model is described in section 2, Basic Reproduction Number in section 3 and Timescale Analysis in section 4. Finally, discussions and conclusion of the analysis are given in sections 5 and 6 , respectively

\section{Dimensionless Model}

We adopt the dimensionless form of the model formulated in [2] as,

$$
\begin{gathered}
\frac{d S_{h}}{d t}=\lambda+\gamma I_{S}+A-\beta I_{m} S_{h} \frac{T_{m}}{N}-\lambda S_{h}+\alpha S_{h} I_{S}+\theta A \\
\frac{d l_{h}}{d t}=\beta I_{m} S_{h} \frac{T_{m}}{N}-\eta L_{h}-\lambda L_{h}+\alpha L_{h} I_{S}+\theta L_{A} \\
\frac{d L_{A}}{d t}=\beta I_{m} A \frac{T_{m}}{N}-\eta L_{A}-\lambda L_{A}+\alpha L_{A} I_{S}-\theta L_{A} \\
\frac{d I_{s}}{d t}=\eta L_{h}+\eta L_{A}-(\alpha+\gamma+\rho+\lambda) I_{S}+\alpha I_{S}^{2} \\
\frac{d A}{d t}=\rho I_{S}-\left(1+\beta I_{m} \frac{T_{m}}{N}+\lambda-\alpha I_{S}+\theta\right) A \\
\frac{d S_{m}}{d t}=q\left(1-S_{m}\right)-b I_{S} S_{m}-d A S_{m}-d L_{A} S_{m}+h I_{m} S_{m} \\
\frac{d L_{m}}{d t}=b I_{S} S_{m}+d A S_{m}+d L_{A} S_{m}-(f+g) L_{m}+h I_{m} L_{m} \\
\frac{d I_{m}}{d t}=f L_{m}-(h+q) I_{m}+h I_{m}^{2}
\end{gathered}
$$

With

$$
d N / d t=-\alpha I_{S} N+(\lambda-\mu) N, d T_{m} / d t=-h I_{m} T_{m}+(q-g) T_{m},
$$

and the model's parameter values defined in Table 1.

Table 1. Dimensionless parameters and their values.

\begin{tabular}{llll}
\hline Dimensional & Dimensionless & Value & Value in $\varepsilon$ \\
\hline$\beta_{h} e T_{m}(0) / l_{a} N(0)$ & $\beta$ & 62.43 & $\mathrm{O}\left(\varepsilon^{-2}\right)$ \\
$\eta_{h} / l_{a}$ & $\eta$ & 11.1 & $\varepsilon^{-1}$ \\
\hline
\end{tabular}

\begin{tabular}{llll}
\hline Dimensional & Dimensionless & Value & Value in $\varepsilon$ \\
\hline$\mu_{h} / l_{a}$ & $\mu$ & 0.0056 & $\mathrm{O}\left(\varepsilon^{2}\right)$ \\
$\beta_{s} e / l_{a}$ & $b$ & 7.2 & $\mathrm{O}\left(\varepsilon^{-1}\right)$ \\
$\beta_{a} e / l_{a}$ & $d$ & 38.2 & $\mathrm{O}\left(\varepsilon^{-1}\right)$ \\
$\lambda_{h} / l_{a}$ & $\lambda$ & 0.017 & $\mathrm{O}\left(\varepsilon^{-1}\right)$ \\
$\alpha_{h} / l_{a}$ & $\alpha$ & 0.01 & $\mathrm{O}\left(\varepsilon^{2}\right)$ \\
$r_{s} / l_{a}$ & $\gamma$ & 11.5 & $\mathrm{O}\left(\varepsilon^{-1}\right)$ \\
$r_{a} / l_{a}$ & $\rho$ & 54.45 & $\mathrm{O}\left(\varepsilon^{-2}\right)$ \\
$\psi \theta_{h} / l_{a}$ & $\theta$ & & \\
$\eta_{m} / l_{a}$ & $f$ & 14 & $\mathrm{O}\left(\varepsilon^{-1}\right)$ \\
$\lambda_{m} / l_{a}$ & $q$ & 21.45 & $\mathrm{O}\left(\varepsilon^{-1}\right)$ \\
$\mu_{m} / l_{a}$ & $g$ & 20.62 & $\mathrm{O}\left(\varepsilon^{-1}\right)$ \\
$\alpha_{m} / l_{a}$ & $h$ & 1.45 & $\mathrm{O}(1)$ \\
\hline
\end{tabular}

By definition, $\varepsilon$ is the ratio of the proportion of time for the latency period $\left(\eta_{h}\right)$ compared to the mean asymptomatic state timescale $\left(l_{a}\right)$. For $\varepsilon \ll 1$, it means that asymptomatic humans remain infectious for a longer time compared to the latency period of humans.

In the adapted model, the human population is divided into compartments of susceptible $\left(S_{h}\right)$, latent $\left(L_{h}\right)$, latent asymptomatic $\left(L_{A}\right)$, symptomatic $\left(I_{s}\right)$ and asymptomatic ( $A$ ) carriers. The mosquito population is subdivided into susceptible $\left(S_{m}\right)$, latent $\left(L_{m}\right)$, and infectious $\left(I_{m}\right)$ compartments. Detailed model description including state variables, schematic flow diagram, and assumption are described in [2]. The rate at which infected mosquitoes bites is $e I_{m}, e$ is a constant value of the biting rate per human per unit time, $\beta_{h}$ is the number of human infections per bite, $\beta_{a}$ being the probability that a bite by a susceptible mosquito on an asymptomatic infectious human transfers the infection to the mosquito, and $\beta_{s}$ depicting the probability that a susceptible mosquito gets infected after biting a symptomatic infectious human.

We also state here that the susceptible mosquitoes are recruited into the mosquito population through a constant birth rate $\lambda_{m}$ with $e T_{m}$ bites by mosquitoes on humans. However, only $S_{h} / N$ of these bites are made on susceptible humans. The proportion that a bite is made by an infectious mosquito is $I_{m} / T_{m} . \beta_{h}$ assumes that not all bites by an infectious mosquito on a susceptible human can lead to infection with the parameter $\beta_{h} \in[0,1]$ depicting the proportion of bites by an infectious mosquito that passes on the infection, where $\beta_{h}=1$ implies all bites transmits the disease.

Furthermore, those in the $L_{h}$ class are already in the process of transition into the $I_{s}$ class who are entitled to treatment. Thus, the incubating humans become infectious after a mean latency time $1 / \eta_{h}$. Also, all humans who die naturally have a per capita rate of $1 / \mu_{h}$, while some 
individual in the $I_{s}$ group die at rate $\alpha_{h} I_{s}$ from the In addition, those who survive receive treatment and are recovered with complete clearance to join the susceptible at a rate $r_{s} I_{s}$ (treatment period 14-days), or only recover from symptoms (after a 3-day mono-therapy) without clearance to join the $A$ class at a rate $r_{a} I_{s}$. The post symptomatic class $A$ still carry merozoites and produce gametocytes. So, they can infect biting mosquitoes. Since patients can be in this state for several weeks or months they can play an important role in sustaining an epidemic. Thus, consider a putative treatment which removes individual from $A$ and $L_{A}$ classes down to $S_{h}$ and $L_{h}$, respectively, with the effect of the treatment parameter being $\psi \theta_{h}$, where $\psi$ are those being treated. Similarly, mosquitoes in the incubating class die naturally at a rate $\mu_{m} L_{m}$ and the rest get infected at a rate $\eta_{m} L_{m}$ to join the infectious class which remain until their death either naturally or are killed by the parasite at rate $\alpha_{m} I_{m}$.

\section{The Basic Reproduction Number}

Since there are no trivial equilibrium points as long as $S_{h}$ and $S_{m}$ are not zero. The implication is that $\left(S_{h}, L_{h}, L_{A}, I_{s}, A, T_{m}, S_{m}, L_{m}, I_{m}\right) \neq(0,0,0,0,0,0,0,0,0) \quad$ and the population is not extinct. Therefore, we define a domain of biological interest for our model in the form

$\Omega=\left\{\left(S_{h}, L_{h}, L_{A}, I_{s}, A, T_{m}, S_{m}, L_{m}, I_{m}, \mathrm{~N}\right) \in \mathbb{R}_{+}^{10} \mid\right.$ they are $\geq 0$ for all $\left.t>0\right\}$, such that the basic reproduction number, $R_{0}$, is determined by the Next Generation Matrix (NGM) method by [2] as

$R_{0}=\frac{\beta\left(b b_{2}+d b_{3}\right) b_{1}}{b_{0}^{2}}=\frac{\beta \eta f(b(1+\lambda+\theta)+\rho d)}{(f+q)(h+q)(\eta+\lambda)(1+\lambda+\theta)(\alpha+\gamma+\rho+\lambda)}$.

The NGM operator approach approximates the number of secondary infections produced by one infected individual and expresses $R_{0}$ as the product of the expected duration of the infectious period and the secondary rate infectious. When $R_{0}<1$, each infected individual produces on average less than one new infected individual so we would expect the disease to die out. On the other hand if $R_{0}>1$, each individual produces more than one new infected individual so we would expect the disease to spread in the population. This implies that the threshold quantity for eradicating the disease is to reduce $R_{0}$ to less than one.

Using equation (9), the stability analysis of the equilibrium in the domain $\Omega$ is attained from the eigenvalues of the Jacobian matrix evaluated at equilibrium point detailed in [2]. The analysis revealed that the disease-free equilibrium is locally and globally stable if $R_{0}<1$ and an endemic equilibrium is not feasible. However, since normally $R_{0} \gg 1$ and the asymptomatic infectious humans to mosquitoes is significantly large, a possible treatment is to reduce the infectivity of the asymptomatic humans, $d$, and the symptomatic humans, $b$ by increasing the parameters $\theta$ and $\lambda$.

\section{Time Scale Analysis}

The asymptotic analysis on $N$ and $T_{m}$ equations depict that $T_{m}$ changes on the time scale $\mathrm{O}(\varepsilon)$ while $N$ changes on $\mathrm{O}\left(\varepsilon^{-2}\right)$. Therefore, an assumption that $T_{m} / N$ is constant over the time scale of the model by setting $\theta=0$ gives,

$$
\begin{gathered}
\varepsilon^{2} \frac{d S_{h}}{d t}=\varepsilon^{4} \hat{\lambda}+\varepsilon \hat{\gamma} I_{S}+\varepsilon^{2} A-\widehat{\beta} I_{m} S_{h}-\varepsilon^{4} \hat{\lambda} S_{h}+\varepsilon^{4} \alpha S_{h} I_{S}, \\
\varepsilon^{2} \frac{d l_{h}}{d t}=\widehat{\beta} I_{m} S_{h}-\varepsilon \hat{\eta} L_{h}-\varepsilon^{4} \hat{\lambda} L_{h}+\varepsilon^{4} \hat{\alpha} L_{h} I_{S}, \\
\varepsilon^{2} \frac{d L_{A}}{d t}=\widehat{\beta} I_{m} A-\varepsilon \hat{\eta} L_{A}-\varepsilon^{4} \hat{\lambda} L_{A}+\varepsilon^{4} \hat{\alpha} L_{A} I_{S},
\end{gathered}
$$

$\varepsilon^{2} \frac{d I_{s}}{d t}=\varepsilon \hat{\eta} L_{h}+\varepsilon \hat{\eta} L_{A}-\left(\varepsilon^{4} \widehat{\alpha}+\varepsilon \hat{\gamma}+\widehat{\rho}+\varepsilon^{4} \widehat{\lambda}\right) I_{S}+\varepsilon^{4} \widehat{\alpha} I_{S}^{2}$,

$$
\varepsilon^{2} \frac{d A}{d t}=\hat{\rho} I_{S}-\left(\varepsilon^{2}+\widehat{\beta} I_{m}+\varepsilon^{4} \widehat{\lambda}-\varepsilon^{4} \widehat{\alpha} I_{S}\right) A
$$

$$
\begin{gathered}
\varepsilon \frac{d S_{m}}{d t}=\hat{q}\left(1-S_{m}\right)-\hat{b} I_{S} S_{m}-\widehat{d} A S_{m}-\hat{d} L_{A} S_{m}+\varepsilon \hat{h} I_{m} S_{m}, \\
\varepsilon \frac{d L_{m}}{d t}=\hat{b} I_{S} S_{m}+\hat{d} A S_{m}+\hat{d} L_{A} S_{m}-(\hat{f}+\widehat{g}) L_{m}+\varepsilon \hat{h} I_{m} L_{m}, \\
\varepsilon \frac{d I_{m}}{d t}=\hat{f} L_{m}-(\varepsilon \hat{h}+\widehat{q}) I_{m}+\varepsilon \hat{h} I_{m}^{2},
\end{gathered}
$$

with initial conditions

$$
\begin{aligned}
& S_{h}(0)=1, \quad L_{h}(0)=0, \quad L_{A}(0)=0, \quad I_{s}(0)=0, \quad \mathrm{~A}(0)=0, \\
& L_{m}(0)=l_{0}, \quad S_{m}(0)=1-l_{0}, \quad I_{m}(0)=0, \quad \varepsilon \ll 1, \quad l_{0} \ll \varepsilon,
\end{aligned}
$$

and the parameters expressed in terms of their size as powers of $\varepsilon$ as follows,

$$
\begin{aligned}
& \beta=\frac{\hat{\beta}}{\varepsilon^{2}}, \quad b=\frac{\hat{b}}{\varepsilon}, \quad d=\frac{\hat{d}}{\varepsilon}, \quad \eta=\frac{\hat{\eta}}{\varepsilon}, \quad \mu=\varepsilon^{2} \widehat{\mu}, \quad \lambda=\varepsilon^{2} \hat{\lambda}, \\
& \alpha=\varepsilon^{2} \hat{\alpha}, \quad \gamma=\frac{\hat{\gamma}}{\varepsilon}, \quad \rho=\frac{\hat{\rho}}{\varepsilon^{2}}, \quad f=\frac{\hat{f}}{\varepsilon}, \quad q=\frac{\hat{q}}{\varepsilon}, \quad g=\frac{\hat{g}}{\varepsilon}, \quad h=\hat{h} .
\end{aligned}
$$

Using singular perturbation method and setting the time scale $t=\varepsilon^{2} \widehat{t}$, we noticed that susceptible humans $\left(S_{h}\right)$ and latent mosquitoes $\left(L_{m}\right)$ are decaying linearly in time from their initial values due to the i) latent mosquitoes converting to the infectious class and ii) susceptible becoming infected as a result of infectious contact with mosquitoes in the $L_{m}$ class. Similarly, by setting $t=\varepsilon^{4 / 3} \widehat{t}$, we observe that all the leading order solutions are the same except that variables $S_{m}$ and $L_{m}$ 
have an additional term, $\widehat{d} \hat{A}_{0}$. This introduces a reaction of infection from asymptomatic class in the susceptible mosquitoes into the susceptible human population. Thus, creating a stability between the amount of mosquitoes converting to the infectious class and the amount becoming infected by biting humans in the asymptomatic infectious class. However, there is a notable difference between $S_{m}$ and $L_{m}$ with an accelerated rate of mosquito infection from asymptomatic infectious humans when the initial conditions $\quad \widehat{S}_{h_{1}}(0)=0, \widehat{L}_{h_{0}}(0)=0, \widehat{L}_{A_{0}}(0)=0, \quad \widehat{I}_{S_{0}}(0)=0$, $\widehat{\mathrm{A}}_{0}(0)=0, \widehat{L}_{m_{1}}(0)=0, \quad \widehat{S}_{m_{1}}(0)=0, \hat{I}_{m_{0}}(0)=0, \quad$ are $\quad$ used. Thus, the flow of the solution may change direction especially when the amount of mosquitoes being infected becomes greater than the inflow of new born mosquitoes.

By setting $t=\varepsilon^{5 / 4} \hat{t}$, we observe that equations (10)-(17) are unchanged. However, due to the dominant contribution of the asymptomatic infectious humans on the infection of mosquitoes, the rate of change of $L_{m_{1}}$ and $S_{m_{1}}$ are proportional to the amount of asymptomatic humans. In addition, $I_{m_{0}}$ is proportional to $L_{m_{1}}$. So, with the following initial conditions

$S_{h_{1}}(0)=0, \quad L_{h_{0}}(0)=0, \quad L_{A_{0}}(0)=0, \quad I_{S_{0}}(0)=0, \quad \mathrm{~A}_{0}(0)=0$,

$L_{m_{1}}(0)=1, \quad S_{m_{1}}(0)=-1, \quad I_{m_{0}}(0)=0, I_{m_{0}}^{\prime}(0)=I_{m_{0}}^{\prime \prime}(0)=I_{m_{0}}^{\prime \prime \prime}(0)=0$,

The time solution for our system equations grow exponentially as follows

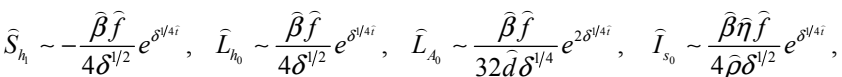

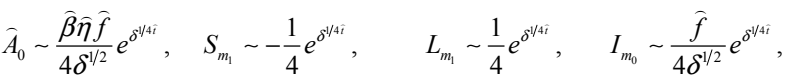

where we have set $\delta=\widehat{\beta} \hat{\eta} \widehat{d} \hat{f}$. If we allow $\delta_{0}=\delta^{1 / 4}$, the approximations for this time-scale is not a good one because $S_{h_{1}}=\mathrm{O}\left(e^{\delta_{0}} \widehat{t}\right)=\mathrm{O}\left(\varepsilon^{1 / 2} / l_{0}\right)$. In other words, when $\widehat{t}=\ln \left(\varepsilon^{1 / 2} / l_{0}\right) / \delta_{0}$, the asymptomatic human becomes infected with new asexual parasites due to contact with infectious mosquitoes.

Now, we set $t=\varepsilon^{\frac{5}{4}} \ln \left(\varepsilon^{1 / 2} / l_{0}\right) / \delta_{0}+\varepsilon^{\frac{5}{4}} \bar{t}$, we are saying that the initial small amount of infection has developed into a full blown epidemic with $S_{h}$ and $L_{h}$ becoming $\mathrm{O}(1)$ and not depending on $l_{0}$. After rescaling our model equations reduces to

$$
\begin{aligned}
& \frac{d \widehat{S}_{h_{0}}}{d \hat{t}}=-\widehat{\beta} \widehat{S}_{h_{0}} I_{m_{0}}, \quad \frac{d \widehat{L}_{h_{0}}}{d \hat{t}}=\widehat{\beta} \widehat{S}_{h_{0}} I_{m_{0}}, \quad \frac{d \widehat{L}_{A_{0}}}{d \widehat{t}}=\widehat{\beta} \widehat{A}_{0} I_{m_{0}}, \quad \frac{d \widehat{I}_{S_{0}}}{d \hat{t}}=\frac{\widehat{\eta}}{\widehat{\rho}} \widehat{L}_{h_{0}}, \\
& \frac{d \widehat{A}_{0}}{d \hat{t}}=\widehat{\rho} \widehat{\rho}_{s_{0}}-\widehat{\beta} \widehat{A}_{0} I_{m_{0}}, \frac{d \widehat{S}_{m_{1}}}{d \hat{t}}=-\widehat{d} \widehat{A}_{0}-\widehat{d} \widehat{L}_{A_{0}}, \frac{d \widehat{L}_{m_{1}}}{d \hat{t}}=\widehat{d} \widehat{A}_{0}+\widehat{d} \widehat{L}_{A_{0}}, \frac{d \widehat{I}_{m_{0}}}{d \hat{t}}=\widehat{f L}_{m_{1}}
\end{aligned}
$$

Here, the asymptomatic humans become infected with new asexual parasites after contact with infectious mosquitoes and eventually reduce the number of the asymptomatic class $(A)$ to the latent asymptomatic class, $\left(L_{A}\right)$. Further examination shows that

$$
\frac{d \widehat{S}_{h_{0}}}{d \hat{t}}+\frac{d \widehat{L}_{h_{0}}}{d \hat{t}}=0, \quad \frac{d\left(\widehat{L}_{A_{0}}+\widehat{A}_{0}\right)}{d \widehat{t}}=\hat{\eta} \widehat{L}_{h_{0}}, \quad \frac{d^{3} \widehat{I}_{m_{0}}}{d \hat{t}^{3}}=\hat{\eta} \widehat{f} \widehat{d} \widehat{L}_{h_{0}} .
$$

In the first equation, $\widehat{S}_{h_{0}}+\widehat{L}_{h_{0}}=1$, replacing this into the $\widehat{S}_{h_{0}}$ equation yields a fourth-order nonlinear equation given by

$$
\frac{d^{4}\left(\ln \widehat{S}_{h_{0}}\right)}{d \bar{t}^{4}}=\frac{d^{4} \widehat{\hbar}}{d \bar{t}^{4}}=-\delta\left(1-e^{\widehat{\hbar}}\right), \quad \text { where } \bar{\hbar}=\ln \left(\widehat{S}_{h_{0}}\right) .
$$

When $\bar{\hbar}=0 \Rightarrow S_{h_{0}}=1$, thus an unstable steady state is achieved. Let consider $c(\bar{\hbar})=-\delta\left(1-e^{\hat{\hbar}}\right)$, then for $\bar{\hbar}<0 \Rightarrow c<0$ and for $\bar{\hbar}>0 \Rightarrow c>0$. As $\bar{t} \rightarrow-\infty$, we have $\bar{\hbar} \rightarrow 0^{-} \Rightarrow S_{h_{0}} \rightarrow 1^{-}$. Hence $d \bar{\hbar} / d \bar{t}<0$ as $\hat{t}$ increases. Thus, a significant amount of humans may become infected. For negatively large $\bar{\hbar}, \frac{d^{4} \bar{\hbar}}{d \bar{t}^{4}} \sim-\delta$ with unresolved constants, $\tau_{i}{ }^{\prime} s$ in the general solution of the form

$$
\widehat{\hbar}=-\frac{1}{24} \delta \widehat{t}^{4}+\frac{1}{6} \tau_{1} \widehat{t}^{3}+\frac{1}{2} \tau_{2} \widehat{t}^{2}+\tau_{3} \widehat{t}+\tau_{4},
$$

Consequently, the solutions for the susceptible and latent human equations become $\widehat{S}_{h_{0}} \sim \bar{\hbar}$ and $L_{h_{0}} \sim 1-\bar{\hbar}$, where $\hat{\hbar}$ is defined above. Thus, showing that the force of infection generated by infectious mosquitoes significantly lowers the size of the susceptible human population $\left(S_{h}\right)$ while increasing that of the latent human $\left(L_{h}\right)$ group. Now, due to the sudden drop in $S_{h}$, there may be series of transition timescale in which $S_{h}=\mathrm{O}(1)$ fall to $S_{h}=\mathrm{O}\left(\varepsilon^{2}\right)$. However, we analyze the main rebalance where the infected mosquito group become significant and the latent group dominate the human population.

Thus, when $t=\varepsilon \hat{t}$ and using the following rescaling variables: $\quad S_{h} \sim \mathcal{E}^{2} \widehat{S}_{h_{0}}, \quad L_{h} \sim \widehat{L}_{h_{0}}$, $L_{A} \sim \widehat{L}_{A_{0}}, I_{s} \sim \varepsilon \widehat{I}_{s_{0}}, A \sim \widehat{A}_{0}, S_{m} \sim \widehat{S}_{m_{0}}, L_{m} \sim \widehat{L}_{m_{0}}, I_{m} \sim \widehat{I}_{m_{0}}$, we notice that the susceptible group is now $\mathrm{O}\left(\varepsilon^{2}\right)$ and that most of human population are in the latent group. Substituting the scaling variables into the original model produces steady state solutions. However, the amount of latent humans decay quickly causing the latent asymptomatic humans to grow as a result of a massive inflow of asymptomatic humans being infected with asexual parasites.

Finally, by setting $t=\varepsilon / \hat{\eta} \ln \left(\mathcal{E}^{-1}\right)+\varepsilon \hat{t} \quad$, $S_{h} \sim \varepsilon^{2} \widehat{S}_{h_{0}}, L_{h} \sim \varepsilon \widehat{L}_{h_{0}}, \quad L_{A} \sim 1, I_{s} \sim \varepsilon \widehat{I}_{s_{0}}$, $A \sim \varepsilon \widehat{A}_{0}, S_{m} \sim \widehat{S}_{m_{0}}, L_{m} \sim \widehat{L}_{m_{0}}, I_{m} \sim \widehat{I}_{m_{0}}, \quad$ and substituting into our model equation, we find that the variables in their 
steady states remain unchanged except $L_{A} \sim 1$. Furthermore, the fraction of latent humans, $L_{h}$ increases as $S_{h}$ decreases. We also observed that $L_{h}$ is no longer $\mathrm{O}(1)$ as $L_{A}$ grows to overtake $L_{h}$.

\section{Discussions}

We have analyzed six main time scales with appropriate rescaling to elucidate the dynamics of malaria disease as they evolve from small infection to endemic state. At $t=\mathrm{O}\left(\varepsilon^{2}\right) \approx 1-3$ days, introducing a small amount of infected mosquitoes into the system and biting susceptible humans causes human to get infected. The early infection indices itself into the population and grows linearly but their effect remains unnoticeable in the latent asymptomatic group.

When $t=\mathrm{O}\left(\varepsilon^{4 / 3}\right) \approx 7-8$ days, the susceptible mosquitoes get infected by biting asymptomatic infectious humans. We noticed that the amount of mosquitoes converting to the infectious group is balanced by the amount of mosquitoes being infected by biting humans in the asymptomatic infectious group. This observation is expected because individuals with clinical malaria have low level of gametocytes. Thus, the early infection of susceptible mosquitoes is likely to come from contact with asymptomatic infectious humans since they have high gametocyte density. The contribution of asymptomatic infectious humans has a significant effect on the dynamics of the disease. Now, as more mosquitoes get infected through contact with asymptomatic infectious population, (i.e. when $t=\mathrm{O}\left(\varepsilon^{5 / 4}\right) \approx 9-10$ days $)$, the amount of susceptible mosquitoes become optimized and starts to decline. However the feedback from infectious humans offsets the linear growth of the initial amount of infected mosquitoes introduced. Thus, causing the amount of latent mosquitoes to grow exponentially.

$$
\text { For } t=\varepsilon^{\frac{5}{4}} \ln \left(\varepsilon^{1 / 2} / l_{0}\right) / \delta_{0}+\varepsilon^{\frac{5}{4}} \hat{t} \text { where } \delta_{0}=(\hat{\beta} \hat{\eta} \widehat{d f})^{1 / 4} \approx 2 \text { weeks, }
$$

the asymptomatic humans become infected with new asexual parasite as a result of contact with infectious mosquitoes. The amount of infected humans to the asymptomatic status is being balanced by the amount of asymptomatic human to the latent asymptomatic group due to a boost in their partial immunity level. Thus, more mosquitoes become infected and the overall distribution of infection results in a fast shift of susceptible humans into the latent group. After this time the disease is now visible with infected humans equal to $\mathrm{O}(1)$. Also, the latent asymptomatic humans may still be infectious to the mosquitoes as $S_{h}, I_{s}$, and $A$ adjust to their equilibrium values and $L_{h}$ decaying exponentially. At approximately two months after the initial introduction of infected mosquitoes, all human classes equilibrate and latent individuals become symptomatic. The mosquito classes also adjust to assume their equilibrium state.

\section{Conclusions}

We completed a detailed six main timescale analysis on the model adapted from [2] with appropriate rescaling to elucidate the dynamics of malaria from small infection to endemic state. In our analysis, $S_{h}$ remain proportional to $L_{h}$, thus showing that the level of the disease depends on the non-immune humans becoming infected. Thus, the buildup of the latent asymptomatic humans at steady state is the main dynamics of malaria in the endemic region. Also, we find that intervention programs may yield better result if implemented before the fourth timescale. During that time, the feedback from infection humans offsets the linear growth effect of the initial amount of infected mosquitoes and this equates to about 2-3 weeks from the initial infection. We also notice that the buildup of the latent asymptomatic humans at steady state is the main dynamics of malaria in the endemic region. This become evident in the time scale of about 1-2 weeks and thus influences the mode of infection in our analysis.

\section{References}

[1] J. D. Charlwood, T. Smith, P. F. Billingsley, W. Takken, E. Lyimo and J. Meuwissen, Survival and infection probabilities of anthropophagic anophelines from an area of high prevalence of Plasmodium falciparum in humans, Bull. Entomol. Res., 87 (1997): pp. 445-453.

[2] K. Annan and C. Mukinay. Stability and Time-Scale Analysis of Malaria Transmission in Human-Mosquito Population, International Journal of Systems Science and Applied Mathematics. Vol. 2, No. 1, 2017, pp. 1-9. doi: 10.11648/j.ijssam.20170201.11.

[3] K. A. Cullen, and Paul M Arguin. "Malaria Surveillance--United States, 2012." Morbidity and Mortality Weekly Report. Surveillance Summaries (Washington, D. C.: 2002) 63.12 (2014): 1-22. MEDLINE with Full Text. Web. 6 Sept. 2015.

[4] M. Dako-Gyeke and H. M. Kofie. "Factors Influencing Prevention and Control of Malaria among Pregnant Women Resident in Urban Slums, Southern Ghana." African Journal of Reproductive Health 19.1 (2015): 44-53. MEDLINE with Full Text. Web. 7 Sept. 2015.

[5] S. Olaniyi, O. S. Obabiyi. Mathematical Model for Malaria Transmission Dynamics in Human and Mosquito Populations with Nonlinear Forces of Infection. IJPAM Vol. 88 No. 1, 2013, 125-156

[6] H. F. Huo, G. M. Qiu. Stability of a Mathematical Model of Malaria Transmission with Relapse. Abs. \& Applied Analysis, Volume 2014.

[7] C. Chiyaka, W. Garira, S. Dube. Transmission Model of Endemic Human Malaria in a Partially Immune Population. Math \& Comp. Modelling 46 (2007) 806-822.

[8] WHO, World Malaria Report 2016. Geneva: World Health Organization; 2016. Licence: CC BY-NC-SA 3.0 IGO.

[9] P. Brown, Trials and tribulations of a malaria vaccine, New Scientist (1991) 18-19.

[10] H. M. Yang, Malaria transmission model for different levels of acquired immunity and temperature dependent parameters (vector). J. Public Health, 34 (2000), 223-231. 
[11] B. Ogutu, A. B. Tiono, M. Makanga, Z. Premji, A. D. Gbado, D Ubben, A. C. Marrast, O. Gaye. Treatment of asymptomatic carriers with artemether-lumefantrine: an opportunity to reduce the burden of malaria. Malaria Journal, [online]. [viewed 09/11/2016].

[12] M. Imwong, K. Stepniewska, R. Tripura, et al. Numerical Distributions of Parasite Densities during Asymptomatic Malaria. JID (2016) 213, 1322-1329.

[13] J. Tumwiine, J. Mugisha, L. Luboobi. A mathematical model for the dynamics of malaria in a human host and mosquito vector with temporary immunity. Journal of Applied Math. \& Computation, Vol. 189 (2005), 1953-1965.

[14] N. Bacaer and C. Sokhna. A reaction-diffusion system modeling the spread of resistance to an antimalarial drug. Math. Biosci. Engrg, Vol. 2 pp. 227-238, 2005.

[15] J. Li, Y. Zhao, S. Li. Fast and slow dynamics of malaria model with relapse. Mathematical Biosci., Vol. 246, No. 1, pp. 94-104, 2013. 